\title{
Les récepteurs des peptides neurodigestifs
}

L'ensemble des fonctions digestives est un processus d'une grande complexité au cours duquel les sécrétions exocrines et endocrines et la motricité intestinale doivent être précisément contrôlées en fonction de l'apport alimentaire. Ce contrôle met en jeu de nombreuses hormones peptidiques dont certaines sont également produites au niveau du système nerveux central où elles participent à la communication entre les cellules nerveuses. Par leur diversité et l'intégration poussée de la régulation de leur sécrétion et de leurs effets, les peptides neurodigestifs représentent un remarquable modèle de signalisation fonctionnelle par l'intermédiaire d'un réseau interconnecté de médiateurs chimiques. Par son intermédiaire sont contrôlés de manière étroitement coordonnée les flux métaboliques, les sécrétions d'ions et d'enzymes, le péristaltisme intestinal et la prolifération cellulaire.

\section{Gabriel Rosselin \\ Directeur de recherche à l'Inserm}

\section{ADRESSE}

G. Rosselin : unité de recherche sur les peptides neurodigestifs et le diabète, Inserm U. 55, centre de recherche Saint-Antoine, 184, rue du faubourg Saint-Antoine, 75012 Paris, France.

\section{TIRÉS A PART}

G. Rosselin : unité de recherche sur les peptides neurodigestifs et le diabète, Inserm U. 55, centre de recherche Saint-Antoine, 184 rue du faubourg Saint-Antoine, 75012 Paris, France. a cohérence des fonctions physiologiques des eucaryotes est due à la réception et à l'intégration par chaque cellule de messages spécifiques provenant de l'environnement, du milieu intérieur et des cellules voisines. Cette spécificité se caractérise par la présence de récepteurs dans les cellules cibles. Les récepteurs des peptides neurodigestifs (PND) sont ainsi un élément clé dans la régulation nutritionnelle. Par leur intermédiaire, l'organisme s'adapte à des situations variables, fonction des disponibilités alimentaires (nature des aliments, quantité, rythme de leur apport, jeûne) et des besoins physiologiques (effort, croissance, grossesse, etc.). Les signaux spécifiques reçus par les cellules digestives et pancréato-hépatiques (DPH) sont élaborés à partir des stimulations provenant des substrats et portant sur des terminaisons neurosensorielles ou sur les cellules endocrines disséminées dans la sphère DPH. L'élaboration post-stimulative des peptides de régulation par les cellules nerveuses ou endocrines va assurer la transmission du signal: directement de cellule à cellule (paracrine), par voie sanguine (hormonale) ou nerveuse (neuro-paracrine ou neurocrine) (Tableau I, page 288). La fonction nutritionnelle (digestion, absorption, stockage et utilisation des substrats) est modulée par interaction directe de ces peptides avec leurs récepteurs: (1) les PND interviennent ainsi sur les échanges hydro-électrolytiques rencontrés tout au long du tube digestif et la sécrétion acide du fundus gastrique; (2) la sécrétion et la synthèse des enzymes digestives; (3) le stockage d'énergie dans le foie et les adipocytes par les sécrétions du pancréas endocrine; (4) la croissance et la différenciation des cellules liées à la fonction nutritionnelle. Du fait du développement considérable des recherches concernant les récepteurs des PND, nous avons restreint la bibliographie à quelques articles parus en 1987 et aux revues spécialisées ayant déjà paru sur ce sujet. Les récepteurs des substances non peptidiques ainsi que les récepteurs liés à la régulation du transit digestif et de la vascularisation DPH ne seront pas examinés ici, de même que les récepteurs des enképhalines, 
surtout présents au niveau des muscles lisses.

Tous les récepteurs indiqués sur le Tableau II ont été caractérisés par l'interaction ligands radiomarquéscellule. Lorsqu'elle est spécifique, la liaison est inhibée de façon compétitive par le PND homologue, alors que les PND hétérologues sont totalement ou partiellement inefficaces. La caractérisation de la liaison a récemment été affinée grâce au progrès de la chimie des peptides, à l'obtention et à la sélection par HPLC (voir abréviations, p. 293) de ligands biologiquement actifs, monoiodés sur un résidu tyrosine défini. La localisation des récepteurs se précise grâce à l'utilisation de trieurs de cellules donnant des popu- lations cellulaires purifiées à partir de tissus hétérogènes, à l'établissement en culture de lignées cellulaires DPH proches des cellules normales et au développement de systèmes informatisés de vidéo-auto-radiographique quantitative permettant l'étude directe du récepteur in situ ([1], Anteunis et al. [2]). La cartographie des récepteurs des $\mathrm{PND}$ présente un grand intérêt en physiologie. (a) La présence d'un récepteur spécifique dans une cellule indique que les fonctions de cette cellule sont directement modulables par le peptide correspondant. (b) Les études cinétiques et stoechiométriques de l'interaction indiquent si le ligand peut opérer aux concentrations auxquelles il est physiologiquement sécrété. (c) L'analyse de la spécificité de la liaison, en indiquant les agonistes d'affinité maximale, définit la vraie nature du récepteur. Par exemple, l'effet du VIP* au niveau des cellules antrales du rat passe par un récepteur de la sécrétine. L'analyse de la spécificité permet aussi le criblage des antagonistes compétitifs occupant le récepteur sans l'activer. Il est ainsi possible de caractériser la séquence et la configuration spatiale qui, au sein d'un ligand sont indispensables à sa liaison et d'analyser la structure supplémentaire requise pour l'activation des récepteurs. (d) Les récepteurs spécifiques ont une distribution caractéristique de

*VIP $=$ vasoactive intestinal peptide

Tableau I

PRINCIPAUX PEPTIDES NEURODIGESTIFS (PND) ET LEURS FONCTIONS AU NIVEAU DES CELLULES DIGESTIVES

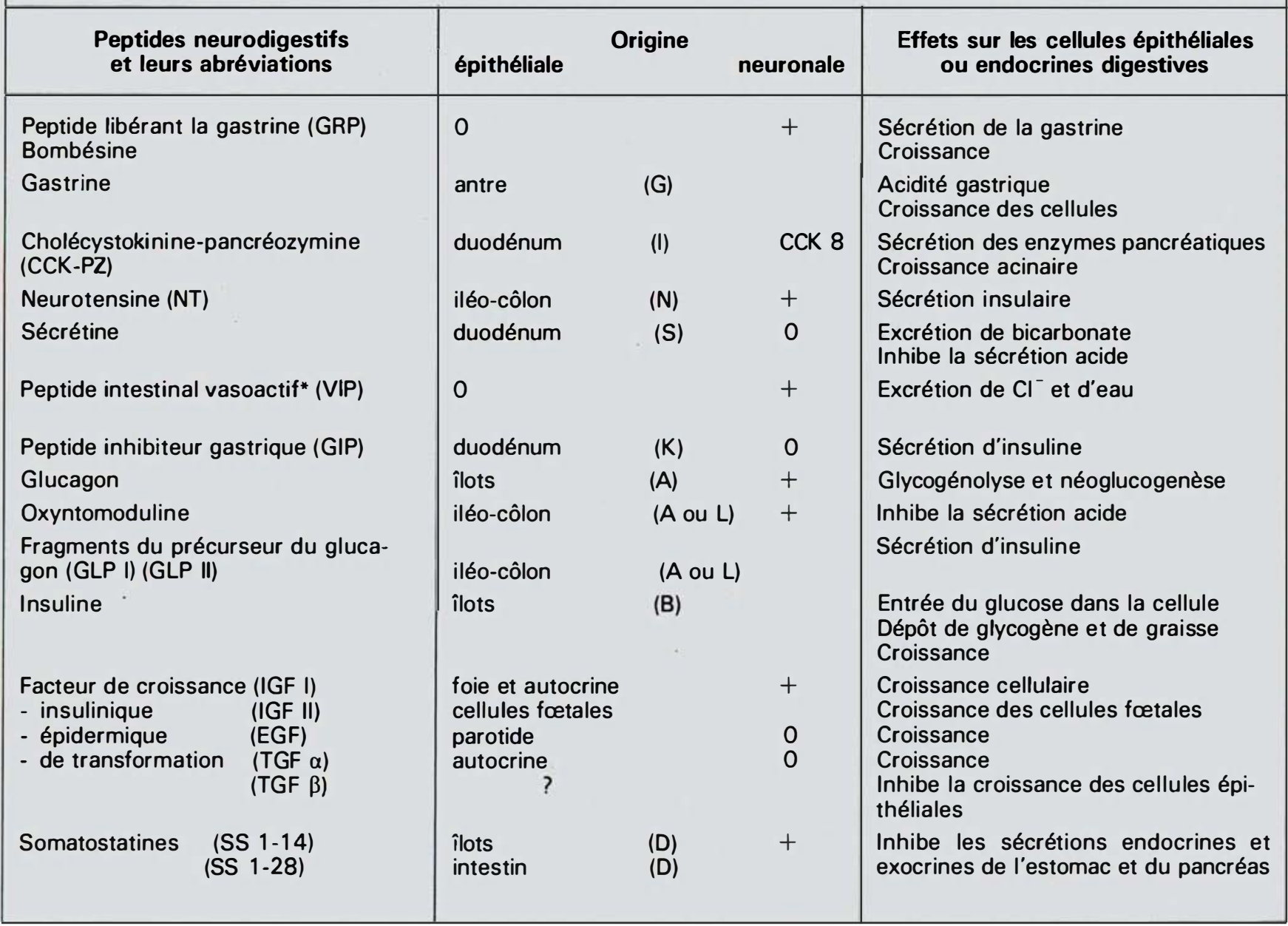

Les lettres entre parenthèses dans la colonne origine indiquent le nom de la cellule sécrétrice.

* Les peptides avec des résidus histidyl N-terminal et isoleucylamide C terminal ou PHI, et celui avec une méthionine C-terminale ou PHM sont rencontrés respectivement chez le rat et l'homme. Ils ont un effet analogue au VIP. 
Tableau II

RÉPARTITION DES RÉCEPTEURS DES PEPTIDES NEURODIGESTIFS (PND) DANS LA SPHĖRE DIGESTIVE ET PANCRÉATICO-HÉPATIQUE

\begin{tabular}{|c|c|c|c|c|c|}
\hline \multirow{3}{*}{ PND } & \multicolumn{5}{|c|}{ Récepteurs confirmés } \\
\hline & \multicolumn{2}{|c|}{ Épithélium } & \multirow{2}{*}{$\begin{array}{c}\text { Acini } \\
\text { pancréatiques }\end{array}$} & \multirow{2}{*}{$\begin{array}{l}\text { Cellule B } \\
\text { des îlots }\end{array}$} & \multirow[t]{2}{*}{ Foie } \\
\hline & gastrique & intestinal & & & \\
\hline Bombésine & * (3) & * (4) & * & * & 0 \\
\hline Gastrine & * (1) & & & & 0 \\
\hline Neurotensine & & * & * & & 0 \\
\hline Sécrétine & $\begin{array}{r}*(2) \\
\text { (rat) }\end{array}$ & 0 & * & & \\
\hline$\left\{\begin{array}{l}\text { VIP, PHI, PHM } \\
\text { Somatocrinine (GRF) }\end{array}\right.$ & $\begin{array}{c}*(2) \\
\text { (homme) }\end{array}$ & * & * & * & * \\
\hline $\begin{array}{l}\text { GIP } \\
\left\{\begin{array}{l}\text { Glucagon } \\
\text { Oxyntomoduline }\end{array}\right.\end{array}$ & * (1) & $\begin{array}{l}0 \\
0 \\
0\end{array}$ & $\begin{array}{l}0 \\
0 \\
0\end{array}$ & $\begin{array}{l}* \\
*\end{array}$ & $\begin{array}{l}0 \\
*\end{array}$ \\
\hline$\left\{\begin{array}{l}\text { Insuline } \\
\text { IGF1 }\end{array}\right.$ & & * & * & * & $\begin{array}{l}* \\
*\end{array}$ \\
\hline EGF (urogastrone) & * (2) & * & * & & * \\
\hline Somatostatine & * (1) & * & * & * & 0 \\
\hline
\end{tabular}

Les peptides neurodigestifs inclus dans une accolade peuvent avoir une réaction croisée au niveau du récepteur, mais seul le récepteur spécifique est indiqué par un astérisque (*). (1): cellule pariétale; (2): cellule à mucus et cellule à pepsine; (3): cellule à gastrine et à somatostatine; (4): cellule à cholécystokinine.

chaque cellule différenciée. Leur étude est donc intéressante en tant que marqueur de différenciation ou pour reconnaître l'origine de cellules tumorales.

Si la séquence des récepteurs de l'insuline, EGF et IGF 1, est maintenant connue (Freychet, Clauser cités dans [3]), la structure moléculaire des récepteurs des autres PND n'est pas encore établie. Les progrès les plus récents dans ce domaine sont dus à la solubilisation des récepteurs du glucagon (Desbuquois cité dans [4]), du VIP [4], de la somatostatine [5, 6], de la CCK $^{*}$ (voir note p. 290) [7], de la bombésine (Linard cité dans [8]) et viendront sans doute de l'obtention d'anticorps monoclonaux spécifiques de ces structures, et de la mise au point de colonnes d'affinité susceptibles de capter le récepteur soluble de façon sélective et réversible. Des preuves indirectes indiquent que les récepteurs des PND sont des glycoprotéines fonctionnellement asymétriques avec trois types de $\mathrm{m} / \mathrm{s} n^{\circ} 5 \mathrm{vol} .4$, mai 88 domaines différents. (1) Le domaine extra-membranaire est dirigé vers l'espace extracellulaire et comporte les sites de reconnaissance. Ces sites sont sensibles aux enzymes protéolytiques et de dégradation des sucres. Le marquage d'affinité utilisan les réactifs bifonctionnels ou l'irradiation UV (ultraviolet) permet de les coupler à leurs ligands spécifiques et de les caractériser par leur migration en gel d'acrylamide SDS (voir abréviations, p.293). (2) Le domaine transmembranaire contient les séquences hydrophobes nécessaires à l'attachement du récepteur à la membrane. (3) Le domaine cytoplasmique inclut les sites effecteurs et régulateurs grâce auxquels l'effet final est transmis à la cellule. L'organisation oligomérique de ces domaines est différente selon les quatre grands types de transduction du signal: le récepteur cholinergique de type nicotinique qui se compose de cinq sousunités se groupant pour former le canal sodium, ne sera pas évoqué ici

puisque ses interactions avec le système peptidergique ne sont pas connues. Les systèmes de reconnaissance des PND sont couplés : (a) à la modulation de la production d'AMP cyclique (récepteurs cyclases); (b) à la stimulation de la phospholipase $\mathrm{C}$; (c) à une activité tyrosine kinase (récepteur-kinases) (figure 1, page 290).

\section{Récepteurs cyclases des PND}

Les PND stimulent (s) ou inhibent (i) la production d'AMP cyclique grâce à un système multimérique qui comporte : (1) les sites de reconnaissance (Rs ou Ri); (2) les protéines de régulation des nucléotides guanyliques (N, G ou G/F); (3) la partie catalytique de l'adénylyl cyclase $(C)$. Les protéines $G$ sont composées de trois sous-unités récemment séquencées : $\alpha \mathrm{i}$ ou $\alpha$ s, $\beta$ et $\gamma$. L'activation de Rs ou Ri par les ligands correspondants provoque la transformation des sous-unités effectrices $\alpha$ s- ou 


\section{RÉFÉRENCES}

1. Rostène WH, Sarrieau A, Moyse E, et al. Imaging of neuropeptide-neurotransmitter interactions. In : de Kloet ER, Wiegant VM, De Wied D, eds. Progress in Brain Research, Vol.22. Amsterdam, New York: Elseviers Sciences Publ, 1987 : 205-11.

2. Said S. Symposium on VIP and related peptides. Annals of the New York Academy of Sciences, New York: 1988.

3. médecine/sciences. In : Freyehet $P$, éd Mécanisme d'action de l'insuline. In : Clauser $E$, ed. Le recepteur de l'insuline, second mes sager de l'hormone. médecine/sciences 1988 $4: 70-1$ et $72-82$

4. Rosselin G. The receptors of the VIP family peptides (VIP, secretin, GRF; PHI PHM, GIP, glucagon and oxyntomodulin). Specificities and identity. Peptides 1986; 7 . 89-100.

5. Lewin MJM. Somatostatin receptors Scand J Gastroenterol 1986; 21 : 42-6.

6. Zeggari M, Viguerie N, Susini C, Garnier $M$, Estève JP, Ribet A. Solubilization and characterization of guinea-pig pancreatic somatostatin receptors. Eur J Biochem 1987 $164: 667-73$

7. Madison LD, Jamieson JD, Rosenzweig SA. Heterogeneity of cholecystokinin receptors in pancreas. Biochem Biophys Res Commun $1987 ; 143$ : 767.

8. Linard C, Reyl-Desmars F, Chen WW Lewin MJM. Solubilization of pancreatic bombesin receptors. International Symposium on Bombesin-like peptides in health and disease. Regul Pept 1987; 19: 99-145 (abstr.).

9. Handbook of physiology. In : Makhlouf $G$, ed. Neuroendocrinology of the gut. In Bataille D, ed. Gut glucagon. In : Rosselin G ed. Liver receptors to regulatory peptides. In Laburthe $M$, ed. Peptide receptors in the intestinal epithelium. Bethesda: Am Phys Soc, 1988 (sous presse).

10. Rosselin G. Physiological relevance of receptor characterization in the study of the entero-insular axis. In : Blazquez E, ed. Frontiers of Hormone Research, Vol.17. Bâle: Karger, 1987.

11. Boissard C, Marie JC, Hejblum G, Gespach C, Rosselin G. Vasoactive intestinal peptide receptor regulation and reversible desensitization in human colonic carcinoma cells in culture. Cancer Res 1986 ; 46 : 4406-13.

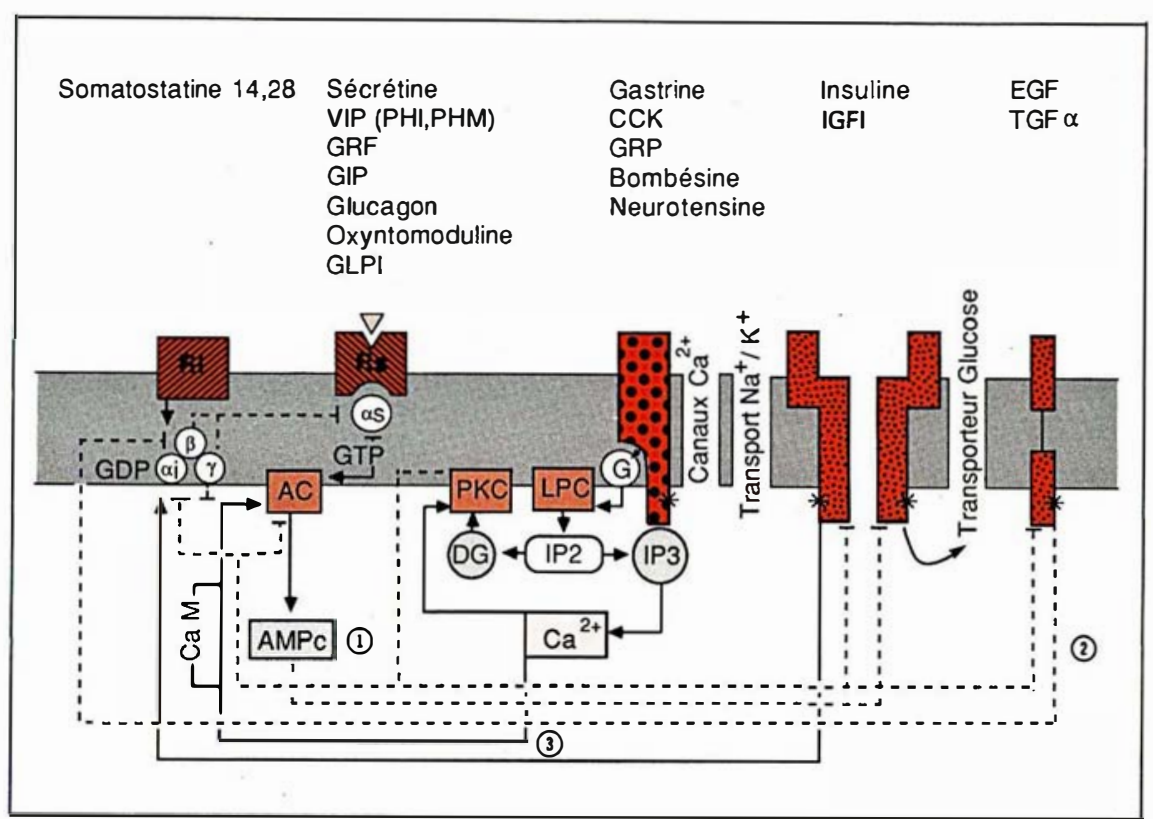

Figure 1. Schéma du réseau des récepteurs-effecteurs des peptides neurodigestifs. Les interactions sont indiquées par des lignes. Les lignes partent du système effecteur et se dirigent vers le système cible soit pour l'activer $(\rightarrow)$ soit pour l'inhiber ( - ) . Le récepteur Rs (rouge hachuré) est en état de stimulation alors que le récepteur Ri (rouge hachuré à la gauche de Rs) n'est pas activé (voir texte). Abréviations: $\alpha i, \alpha s, \beta, \gamma=$ trimères de la protéine $G ; A C=$ site catalytique de l'adénylyl cyclase ; PKC = phosphoprotéine kinase $C ; P L C=$ phospholipase $C ;$; = diacylglycérol; * = récepteur tyrosine kinase (le récepteur à la bombésine a aussi une fonction tyrosine-kinase); Ri, Rs, IP2, IP3, voir texte; $G=$ protéine $G$; CaM = calmoduline. Les interactions représentées sont les suivantes: (1) phosphorylation d'AC, de $G$, des récepteurs par la kinase $C$; (2) interaction des récepteur-kinases avec $\mathrm{G}$ et $\mathrm{AC}$; (3) interaction $\mathrm{Ca}^{2+}$ et cyclase par l'intermédiaire de la calmoduline. Le détail des interactions du récepteur insuline avec les autres systèmes n'est pas indiqué ici. Le lecteur peut se reporter à la référence [3] et à la revue de J. Espinal (Nature 1987; 328: 574-5). VIP = vasoactive intestinal peptide; PHI, PHM = peptides histidyl isoleucylamide et histidyl méthionine; GRF $=$ somatocrinine $;$ GIP = gastric inhibitor peptide $; G L P I=$ fragment 1 du précurseur du glucagon; $C C K=$ cholécystokinine $; G R P=$ gastrin releasing peptide ; IGFI = insulin-like growth factor l; EGF = epidermal growth factor; TGF $\alpha=$ transforming growth factor.

$\alpha \mathrm{i}-\mathrm{GDP}$ en $\alpha \mathrm{s}$ - ou $\alpha \mathrm{i}-\mathrm{GTP}$ qui se dissocient alors des sous-unités $\beta, \gamma$ et agissent sur l'unité catalytique. L'étude fonctionnelle de ce système a été jusque-là aidée par des toxines bloquant $\alpha \mathrm{i}$ (toxine de Bordetella pertussis) ou stimulant $\alpha$ s (toxine cholérique) ou par la forskoline (diterpène activant la sous-unité catalytique $\mathrm{C}$ ). Le séquençage de ces sous-unités rend désormais possible la caractérisation immunologique directe de chacun de ces composés. A noter que les sous-unités $\beta, \gamma$ sont identiques pour les systèmes de stimulation ou d'inactivation et représentent ainsi un élément d'intégration du signal dans les cellules possédant $\mathrm{Rs}$ et $\mathrm{Ri}$. Les récepteurs des peptides neurodigestifs stimulant ou inhibant la production
d'AMP cyclique ont fait l'objet de revues récentes $[4,5](D$. Bataille [9] et éditorial de ce numéro, p. 272).

- Les sites de liaison de l'oxyntomoduline ont été caractérisés au niveau du fundus gastrique qui contient les glandes oxyntiques. Ces glandes occupent la plus grande surface de la niuqueuse gastrique et sont caractérisées par la présence de cellules pariétales ou oxyntiques ainsi que de cellules principales (chief cells) sécrétant la pepsine, et de cellules à mucus. L'oxyntomoduline est ainsi nommée parce qu'elle inhibe la sécrétion acide des cellules pariétales présentes dans les glandes oxyntiques du fundus gastrique et que son mécanisme d'action est paradoxal. En effet, elle stimule de façon similaire à l'histamine (stimulateur de 
l'acidité gastrique), la production d'AMP cyclique dans les cellules fundiques (Bataille [9] et éditorial de ce numéro, p. 272). L'oxyntomoduline ainsi que le GLPl (1) stimulent la sécrétion de l'insuline dépendant de l'AMPc (Bataille cité dans [9], Holst cité dans [10]).

- Les éléments nouveaux concernant le récepteur du VIP présent dans les différentes structures digestives (cellules à mucus et à pepsine de l'estomac humain et de cobaye, cellules épithéliales digestives de la vésicule, du petit intestin et du côlon, cellules des muscles lisses du tube digestif et des vaisseaux, cellules acinaires pancréatiques, cellules hépatiques) font l'objet d'un livre actuellement sous presse[2] et concernent les rapports entre l'activation du récepteur et les échanges hydro-électrolytiques qu'il régule, en particulier la sécrétion d'ions chlore (Dharmsathaphorn cité dans [2]) ainsi que les progrès relatifs à la solubilisation du récepteur (Sudhir et al., O'Doresia et al., Laburthe et al. [2] et Nguyen cité dans [4]), son internalisation [11] et son développement ontogénique [12]. Le rôle du $\mathrm{PHI}^{(2)}$ (ou du $\mathrm{PHM}^{(2)}$ chez l'homme) dont le gène est commun à celui du VIP mais transcrit différemment, apparaît analogue à celui du VIP ; cependant le PHI n'a pas de récepteur propre et a une affinité au moins dix fois plus faible que le VIP pour les récepteurs VIP. La sécrétine a un récepteur spécifique dans les acini pancréatiques et spécifiquement chez le rat dans les cellules épithéliales à mucus et dans les cellules pepsiques (Gespach cité dans [4]); son affinité pour les récepteurs du VIP est plus faible que celle du PHI.

- Le GIP(3) présente des sites de liaison spécifiques au niveau des cellules $\beta$ de hamster et humaines, comme récemment démontré à partir de membranes plasmiques d'un adé-

\footnotetext{
1 GLPI : fragment 1 du glucagon.

2 PHI, PHM: peptides histidyl-isoleucylamide et histidyl-méthionine (voir Tableau I). 3 GIP : gastric inhibitory peptide.

4 GRF : growth hormone releasing factor (somatocrinine).

5 rGRF: GRF de rat.

6 GRP : gastrin releasing peptide.

7 CCK $=$ cholécystokinine.

$\mathrm{m} / \mathrm{s} n^{\circ} 5$ vol. 4, mai 88
}

nome langerhansien sécrétant l'insuline sous l'effet du GIP [13]. Une observation récente suggère la présence de sites récepteurs pour le $\mathrm{GRF}^{(4)}$ dans les cellules $\beta$ (Green $e t$ $a l$. et Hermausen et al. cités dans [10]).

- Citons parmi les antagonistes des PND couplés à la stimulation de l'AMP cyclique, les molécules où le résidu histidyl $\mathrm{N}$-terminal (commun au glucagon, au VIP, à la sécrétine et au rGRF ${ }^{(5)}$ ) est supprimé, modifié ou remplacé, ainsi du N-acétyl-tyr, D-phé, GRF-1-29 $\mathrm{NH}_{2}$ (Robberecht cité dans [4]) antagoniste du VIP. Le des-His ${ }^{1}-\left[\mathrm{Glu}^{9}\right]$ glucagon amide [14] est un bon antagoniste de l'activité cyclasique du glucagon. Le mécanisme d'action des ligands couplés à $\mathrm{Ri}$ (somatostatine) sera évoqué plus loin.

\section{Récepteurs des PND couplés à la PLC et à I'activation de la PKC}

Ce système, revu par ailleurs (Hokin cité dans [10]) est opérationnel pour les PND indiqués dans la figure 1, ainsi que pour les récepteurs cholinergiques de type muscarinique. Ils sont couplés à des effets à court terme et à long terme.

- Au niveau des acini, le système PLC-PKC est activé par la $\operatorname{CCK}^{(7)}$ et couplé à la sécrétion enzymatique (détectable par la sortie d'amylase) ainsi qu'à l'activité des transports électrolytiques $\mathrm{Na}^{+} / \mathrm{K}^{+}[15]$. La CCK-8 (neurocrine) et les neuromédiateurs tels que les neuromédines $B$ et $C$, la bombésine et le GRP. $27^{(6)}$ [16] sont aussi efficaces que la CCK-33 (endocrine) soulignant l'importance de cette fonction. La neurotensine et ses fragments natifs NT 1-8 et 1-1l sont également efficaces sur les acini à des concentrations aussi faibles que $10^{-15} \mathrm{M}$. Au niveau de l'estomac l'activation du récepteur des cellules pariétales (et de la sécrétion acide) par la gastrine passe aussi par le système $\mathrm{IP}_{3}$. Des récepteurs spécifiques de la CCK ou de la bombésine [17] sont détectables au niveau des îlots pancréatiques ou dans des cellules $\beta$ en culture. Les cellules $\mathrm{D}$ du fundus gastrique, sécrétant la somatostatine, ont un système protéine kinase $\mathrm{C}$ sensible à la CCK et à la gastrine [18]. L'effet CCK est

supporté par sept résidus aminés Cterminaux et l'effet gastrine par les cinq résidus aminés C-terminaux communs avec ceux de la CCK. Les antagonistes du récepteur CCK comportent des analogues peptidiques obtenus par modification des sept résidus aminés C-terminaux de la CCK [19] ou des substances non peptidiques, tels les analogues du proglumide connus pour leur propriété anti-acide, le dibutyryl-GMP et les dérivés de l'aspercilin qui contiennent une structure active analogue de la 1-4 benzodiazépine connue pour ses propriétés anxiolytiques. Ces antagonistes sont compétitifs à des degrés divers des liaisons de la CCK au niveau des acini, du cerveau, ainsi que des liaisons de la gastrine au niveau de l'épithélium fundique. Ils sont donc très utiles pour caractériser de façon différentielle les récepteurs périphériques et centraux (Evans et al., Chang et al., Hahn et al. cités dans [19]).

$\mathrm{Au}$ niveau du foie, les récepteurs couplés au système $\mathrm{IP}_{3}$ sont bien différents incluant des peptides sécrétés hors de la sphère digestive, telle la lysine-vasopressine ou probablement synthétisés dans le lit vasculaire hépatique, telle l'angiotensine (Rosselin cité dans [9]).

- L'effet direct sur la croissance des PND activant la kinase $C$ a été constaté pour la gastrine, la cholécystokinine et la bombésine. La pentagastrine et surtout la gastrine-17 stimulent la synthèse d'ADN dans les glandes oxyntiques. La gastrine stimule la croissance de tumeurs du tractus gastro-intestinal, transplantées ou chimiquement induites et il a été démontré que la gastrine-17 à faible concentration (nM) stimule directement la croissance d'une lignée cellulaire de cancers coliques humains (LoVo) une fois ces cellules synchronisées par la thymidine [20]. Alors que la gastrine agit peu sur le pancréas, la cholécystokinine stimule la croissance des cellules pancréatiques, duodénales et de la vésicule et peut-être du petit intestin, mais son rôle physiologique est discuté. La bombésine stimule la croissance de cellules pancréatiques cancéreuses, type AR42J par l'intermédiaire d'un récepteur spécifique [21] et a des effets généraux sur 


\section{RÉFÉRENCES}

12. Chastre E, Emami S, Rosselin G, Gespach C. Ontogenic development of vasoactif intestinal peptide receptors in rat intestinal cells and liver. Endocrinology 1987; 121 : 2211-21

13. Maletti M, Altman JJ, Hui Bon Hoa D, Carlquist M, Rosselin G. Evidence of functional gastric inhibitory polypeptide (GIP) receptors in human insulinoma. Diabetes 1987 ; 36 : 1336-40.

14. Unson CG, Andreu D, Gurzenda EM, Merrifield RB. Synthetic peptide antagonists of glucagon. Proc Natl Acad Sci USA 1987 ; $84: 4083-78$

15. Hootman SR, Brown ME, Williams JA. Phorbol esters and A23187 regulate $\mathrm{Na}^{+}-\mathrm{K}^{+}$. pump activity in pancreatic acinar cells. $\mathrm{Am}$ J Physiol 1987 ; 252 : G499-505.

16. Otsuki M, Fujii M, Nakamura T, et al. Effects of neuromedin $\mathrm{B}$ and neuromedin $\mathrm{C}$ on exocrine and endocrine rat pancreas. $\mathrm{Am}$ J Physiol 1987 ; 252 : G491-8.

17. Swope SL, Schonbrunn A. Characterization of ligand binding and processing by bombesin receptors in an insulin-secreting cell line. Biochem J 1987 ; 247 : 731-8.

18. Chiba T, Syugano K, Park J, Yamada T. Potential mediation of somatostatin secretion from canine fundic D-cells by protein kinase C. Am J Physiol 1987 ; 253 : G62-7.

19. Lignon MF, Galas MC, Rodriguez M, Laur J, Aumelas A, Martinez J. A synthetic peptide derivative that is a cholecystokinin receptor antagonist. J Biol Chem 1987 ; 262 : 7226-31.

20. Kusyk CJ, McNiel NO, Johnson LR. Stimulation of growth of a colon cancer cell line by gastrin. Am J Physiol 1986; 251: G597-601.

21. Logsdon CD, Zhang J, Guthrie J, Vigna $\mathrm{S}$, Williams JA. Bombesin binding and biological effects on pancreatic acinar AR42J cells. Biochem Biophys Res Commun 1987; $144: 463-8$.

22. Fukuoka S, Fushiki T, Kitagawa Y, Sugimoto E, Iwai K. Competition of a growth stimulating/cholecystokinin (CCK) releasing-peptide (monitor peptide) with epidermal growth factor for binding to 3TY3 fibroblasts. Biochem Biophys Res Commun 1987; $145: 646-50$

23. Trimble ER, Bruzzone $R$, Biden T J, Meehan CJ, Andreu D, Merrifield RB. Secretion stimulates cyclic AMP and inositol triphosphate production in rat pancreatic acinar tissue by two fully independent mechanisms. Proc Natl Acad Sci USA 1987; 84 : 3146-50.

24. Kurokowa M, Lynch K, Podolsky DK. Effects of growth factors on an intestinal epithelial cell line : transforming growth factor $\beta$ inhibits proliferation and stimulates differentiation. Biochem Biophys Res Commun $1987 ; 142: 775-82$. la croissance du tractus gastrointestinal et du pancréas chez le nouveauné (Lehy cité dans [10]). La bombésine agit-elle sur la croissance des cellules digestives par l'intermédiaire d'une protéine $G$ en partie inhibable par la toxine de Bordetella pertussis ? Aucune expérience ne permet actuellement de l'affirmer.

\section{Récepteur-kinases des PND}

Ces peptides, à des degrés divers, présentent des homologies : d'origine (le système DPH), de structure, de récepteur et de fonction. Ils comportent l'insuline sécrétée par les cellules $\beta$ du pancréas endocrine, les facteurs de croissance insulino-semblable, IGF I ou somatomédine $\mathrm{C}$ produit par le foie, et IGF II analogue au facteur d'origine hépatique stimulant l'activité de mélanocytes (MSA). L'autre famille de facteurs de croissance comporte l'EGF, abondant dans la parotide et identique à l'urogastrone, l' $\alpha$-TGF (analogue pour les 19 résidus $\mathrm{N}$-terminaux) et codé par un gène différent de celui de l'EGF. Le monitor peptide [22] a été récemment détecté dans les sécrétions pancréato-biliaires; ses résidus aminés 20-33 sont analogues à l'EGF 518 de souris. L'insuline et l'EGF ont des récepteurs dans différentes cellules du système GPH (Tableau II). Les récepteurs de l'insuline sont couplés à la synthèse d'ARNm ou de protéine (Williams et al., Kobari et al., Bréant et al. cités dans [10]) dans l'épithélium digestif, voire à la stimulation de la duplication du noyau dans les cellules cancéreuses coliques en culture (César cité dans [10]). Malgré la communauté d'action de l'insuline et de l'IGF I sur la croissance, il existe de grandes différences dans leurs fonctions, explicables à plusieurs niveaux. L'insuline en tant qu'hormone est un point-clé de la coordination entre l'apport alimentaire, l'utilisation du glucose et la régulation de la croissance. Elle agit sur la croissance sinon directement par son récepteur, du moins à forte concentration par l'intermédiaire du récepteur de l'IGF I. L'IGF I dont la production hépatique est réglée par l'hormone de croissance et en partie par l'insuline ne circule que sous forme liée à des protéines ce qui module son action hormonale immédiate et majore ses effets autocrines ou paracrines. Quant aux récepteurs de type II qui lient préférentiellement l'IGF II, leur rôle dans la croissance, certain à l'âge foetal, n'est pas démontré dans le système DPH normal de l'adulte.

\section{Récepteurs et filtration membranaire des messages}

La notion simple qu'une réponse cellulaire déterminée est directement liée à l'activation d'un récepteur par un ligand n'est plus valable. En effet: (1) une molécule peptidique peut activer des récepteurs différents ; (2) les molécules de récepteur-effecteur peuvent être connectées entre elles par l'intermédiaire de sites spécifiques formant dans la membrane plasmique un réseau fonctionnel de régulation (figure 1 ). De plus, le même récepteur peut être couplé à des effecteurs différents. Ainsi des effets à court terme (transport, sécrétion enzymatique) et à long terme (multiplication cellulaire) sont transmis par l'intermédiaire du même récepteur (effet de la gastrine, de la cholécystokinine, de la bombésine, de l'insuline ou de l'IGF I).

- Un PND peut activer plus d'un récepteur. Il est bien connu que les récepteurs du glucagon au niveau du foie et de la sécrétine au niveau des acini pancréatiques sont couplés à la stimulation d'adénylate cyclase. Ces deux peptides, dans certaines conditions, peuvent aussi stimuler d'autres systèmes effecteurs et ceci semble-t-il par un ou des récepteurs différents. La secrétine agit également sur IP3 et cet effet dépend d'une structure peptidique différente de celle qui détermine l'effet cyclase puisque la substitution de la leucine par la tyrosine en 10 et en 13 de la sécrétine diminue l'effet PIP3 sans modifier l'effet AMPc [23].

Dans le cas du glucagon la voie PLC serait stimulée à des concentrations plus faibles que celles qui sont nécessaires pour activer la cyclase (Wakelam cité par Bataille dans [9]). Il a de plus été récemment démontré que le clivage d'un peptide de régulation par des peptidases membranaires 
peut engendrer des dérivés agissant sur des récepteurs différents de celui du peptide initial. Ainsi le glucagon 19-29 qui est totalement inefficace pour activer la cyclase inhibe le transport $\mathrm{Ca}^{2+}$ dans les membranes plasmiques de foie avec une efficacité 1000 fois plus grande que celle du glucagon (Mallat et al. et Bataille cités dans [9]). Le clivage tryptique du GRP 1-27 donne des fragments type GRP 14-27 ou 18-27 qui ont une affinité différente de celle du GRP 127 pour les cellules sécrétant la gastrine ou la somatostatine.

- L'intégration des signaux passant par les récepteurs survient déjà dans la membrane cellulaire.

Les PND à fonction inhibitrice. Ils ne seront qu'évoqués ici, qu'il s'agisse du polypeptide pancréatique, inhibiteur de la sécrétion pancréatique, du peptide YY (PYY), inhibiteur des sécrétions acide et pancréatique, du peptide en rapport avec le gène de la calcitonine (CGRP), inhibiteur de la sécrétion acide, de la galanine, inhibiteur de la sécrétion insulinique et des sécrétions pancréatiques, du TGF $\beta$,

\section{${ }^{*} A B R E ́ V I A T I O N S^{*}$}

DPH : cellules digestives et pancréato-hépatiques.

HPLC: high pressure liquid chromatography.

PLC : phospholipase C.

$\boldsymbol{P I P}_{2}$ : phosphatidyl-inositol-4, 5diphosphate.

IP $_{3}$ : myoinositol-1, 4, 5-triphosphate.

PKC : phosphokinase $C$.

GTP: guanosine triphosphate.

SDS: sodium dodecyl sulfate. Détergent utilisé pour supprimer la structure tertiaire des peptides et comparer leur vitesse de migration électrophorétique.

Les abréviations des peptides neurodigestifs sont données dans le tableau I et répondent aux abréviations de la terminologie anglaise. La lettre minuscule parfois présente devant les majuscules correspond à l'espèce. Par exemple, rGRF : rat growth hormone releasing factor. GIP : gastric inhibitory peptide. GRP: gastrin releasing peptide. GLP: glucagon like peptide (I ou II). Les significations de PHI et PHM sont indiquées en note du tableau I. inhibiteur de croissance des cellules épithéliales [24]. En effet, quoique l'existence de liaison spécifique ait été décrite pour la galanine et le PYY (Laburthe cité dans [9]), le mécanisme de l'inhibition reste encore inconnu. Le mécanisme d'action de la somatostatine est mieux connu. L'activation du récepteur des somatostatines S-14 ou S-28 détermine à la fois une diminution de l'activité cyclasique par l'intermédiaire de la protéine Gi (effet supprimé par la toxine de Bordetella pertussis) et une inhibition de la translocation calcique provoquée par le système IP3 [5]. Il existe d'ailleurs de nombreux exemples d'interactions entre $\mathrm{Ca}^{2+}$ et le système AMP cyclique. L'augmentation de la concentration cellulaire du calcium s'associe à un accroissement des taux d'AMP cyclique au niveau des cellules principales de l'estomac, de l'épithélium intestinal ou des cellules $\beta$ par activation cyclasique suivant un système calmoduline-dépendant puisqu'il est inhibé par la trifluoropérazine (figure 1). La possibilité d'une interaction directe des sous-unités $\beta, \gamma$ de la cyclase avec la calmoduline a été récemment démontrée dans le cerveau[25]. La protéine kinase $\mathrm{C}$ en interagissant avec des monomères ou des sousunités de récepteurs module leur activité par l'intermédiaire de phosphorylations spécifiques. Les phosphorylations induites par l'activation de la PKC concernent: (1) la sous-unité catalytique de la cyclase [26]; (2) la sous-unité $\alpha$ de $\mathrm{Gi}$ [25] supprimant ainsi sa fonction d'inhibiteur de la cyclase; (3) le récepteur de l'EGF au niveau de la thréonine; (4) celui de l'insuline au niveau de la sérine; (5) celui de l'IGF 2. L'activation de la PKC provoque une diminution de l'affinité du récepteur de l'EGF alors que son effet sur le récepteur de l'insuline est différent: elle le phosphoryle directement et réduit son activité protéine-tyrosine kinase (Bollag cité dans[27]). Ce type d'inhibition du récepteur de l'insuline reproduit les caractéristiques biochimiques rencontrées chez les dia bétiques avec insulino-résistance.

Les rapports entre le système AMP cyclique et le récepteur de l'insuline sont également intéressants de par leurs conséquences en diabétologie.
Ainsi chez les rats rendus insulinoprives par une intoxication à la streptozotocine* (voir note p. 294), l'activation de l'adénylyl cyclase du foie par le glucagon est accru alors que la liaison du glucagon est analogue à celle d'animaux contrôles (Rosselin [9]). Les taux de protéine $\mathrm{Gi}$, diminués par la carence insulinique, retournent à la normale après administration d'insuline [25, 26]. La sousunité de Gi peut aussi être un substrat de la tyrosine kinase du récepteur de l'insuline. Enfin, l'activité de l'insuline peut être inhibée par l'activation de la sous-unité catalytique de la protéine kinase AMPc-dépendante (Freychet cité dans [3]).

Les rapports récepteurs-substrats sont connus de longue date au niveau de la cellule $\beta$. Le glucose potentialise l'effet du système AMP cyclique [10]. De plus, il favorise la mobilisation calcique par inhibition de l'IP3-5phosphomonoestérase [28] couplant ainsi les systèmes récepteurs des PND aux variations du substrat physiologique le plus important de l'insulinosécrétion.

De l'annonce faite à la membrane à la duplication du noyau. L'identification du réseau fonctionnel des récepteurs PND contrôlant la croissance et la multiplication des cellules digestives est une étape importante dans la compréhension de nombreux phénomènes dont le mécanisme reste jusque-là inconnu: (1) la population de cellules épithéliales dans la muqueuse intestinale se situe dans un continuum dynamique allant des cellules souches à prolifération active aux entérocytes définitivement différenciés sans aucune activité mitotique. La déperdition continue de cellules intestinales est ainsi compensée de façon équilibrée par leur prolifération dans les cryptes actives et leur maturation en cellules absorptives fonctionnelles suivant un cycle de 24-48 heures. La rapidité de ce cycle apparaît étroitement dépendre des apports alimentaires; (2) la régénération des cellules de l'épithélium digestif ou des hépato. cytes après résection chirurgicale; (3) enfin les cancers digestifs (estomac, côlon, pancréas) ont le triste privilège d'être les plus fréquents de tous les cancers. Une augmentation de la réplication cellulaire et l'accroissement du compartiment proli- 


\section{RÉFÉRENCES}

25. Katada T, Kusakabe K, Oinuma M, Ui M. A novel mechanism for the inhibition of adenylate cyclase via inhibitory GTP-binding proteins. J Biol Chem 1987 ; 262 : 11897-900.

26. Yoshimasa T, Sibley DR, Bouvier RJ, Lefkowitz J, Caron MG. Cross-talk between cellular signalling pathways suggested by phorbol-ester-induced adenylate cyclase phosphorylation. Nature 1987 ; 327 : 67-70.

27. Pennington SR. G proteins and diabetes. Nature 1987 ; 327 : 188-9.

28. Rana RS, Sekar MC, Mertz RJ, Hokin LE, MacDonald MJ. Potentiation by glucose metabolites of inositol triphosphate-induced calcium mobilization in permeabilized rat pancreatic islets. J Biol Chem 1987; 262 : 13567-70.

29. Terpstra OT, Van Blankenstein M, Dees J, Eilers GA. Abnormal pattern of cell proliferation in the entire colonic mucosa of patients with colon adenoma or cancer. Gas troenterology 1987 ; 92 : 704-8.

30. Meītzer SJ, Ahnen DJ, Battifora $\mathrm{H}$, Yokota J. Protooncogene abnormalities in colon cancers and adenomatous polyps. Gas. troenterology $1987 ; 92$ : 1174-80.

31. Chesa PG, Rettig WJ, Melamed MR, Old L J, Niman HL. Expression of p2l ras in normal and malignant human tissues : Lack of association with proliferation and malignancy. Proc Natl Acad Sci USA 1987 ; 48 : 3234-8.

32. Ruff MR, Martin BM, Ginns EI, Farrar WL, Pert CB. CD4 recptor binding peptides that block HIV infectivity cause human monocyte chemotaxis. FEBS Lett, $1987 ; 211$ : 17-22.

33. Honda $T$, Adachi $H$, Noguchi $M$, et al. Carbachol regulates cholecystokinin receptor on pancreatic acinar cells. Am J Physiol 1987 ; 252 : G77-83.

34. Meda P, Bruzzone R, Chanson M, Bosco D, Orci L. Gap junctional coupling modulates secretion of exocrine pancreas. Proc Natl Acad Sci USA 1987; 84 : 4901-4.

35. Griffen SC, Russel SM, Katz LS, Nicoll CS. Insulin exerts metabolic and growthpromoting effects by a direct action on the liver in vivo: clarification of the functional significance of the portal vascular link between the beta cells of the pancreatic islets and the liver. Proc Natl Acad Sci USA 1987; 84 : 7300-4.

36. Smith JJ, Derynck R, Korc M. Production of transforming growth factor in human pancreatic cancer cells : evidence for a superagonist autocrine cycle. Proc Natl Acad Sci USA $1987 ; 84$ : 7567-70.

* Toxique spécifique, aux doses utilisées, des cellules $\beta$ des îlots de Langerhans. fératif est un facteur de risque du cancer [29].

- Alors que les données concernant la régulation de la croissance normale des cellules digestives sont inexistantes, il apparaît que la transformation cancéreuse est corrélée à la modification ou la dérégulation de gènes («oncogènes») dont certains codent pour des protéines intervenant dans la transmission des signaux cellulaires décrits ci-dessus. Ces données ont fait rechercher activement des anomalies dans les cellules cancéreuses digestives susceptibles d'expliquer leur déréglement par: (1) l'apparition ou l'accroissement de la sécrétion de facteurs de croissance autocrines (exemple: le TGF $\alpha$ ); (2) la surexpression des récepteurs aux facteurs de croissance et/ou d'oncogènes $(c-m y c$ [30], $c$-erb $B, c-m y b$ ) (Alitalo et al. cités dans [30]) ; (3) des altérations génomiques au niveau des oncogènes susceptibles de donner une protéine de régulation de la croissance qui serait en permanence activée (exemple: l'expression d'un récepteur à l'EGF anormal) (Yamamoto et al., Fukushima et al., Mc Coy et al. cités dans [30]) ou les mutations de l'oncogène ras (Lacal cité dans[30]). Cependant ces anomalies ne sont rencontrées que dans une proportion relativement faible de cancers, et ne sont pas toujours corrélées à la rapidité proliférative cancéreuse [31] sans doute parce que le développement d'une prolifération tumorale digestive implique plusieurs, plutôt qu'une seule modification des gènes régulant la croissance.

- Enfin les mécanismes de reconnaissance des PND peuvent être impliqués dans l'attachement initial d'enveloppes virales. Le virus de la vaccine se lie au récepteur EGF (Epstein cité dans [32]) et un peptide présent dans la coque protéique du virus du SIDA [32] est en partie analogue de la séquence 7-11 (TDNYT) du VIP.

\section{Régulation cellulaire des récepteurs des PND}

Les cellules internalisent leurs récepteurs soit de façon constitutive, soit après l'activation du récepteur par le ligand. Le processus d'internalisation ou d'endocytose décrit pour cer- tains PND (Rosselin et al., cités dans [2]) a les fonctions suivantes : il règle le nombre des récepteurs de surface ; ainsi le traitement préalable des cellules par l'insuline, le glucagon, le VIP, la cholécystokinine, induit une diminution de la densité de leurs récepteurs spécifiques à la surface cellulaire et ainsi diminue l'impact d'une stimulation subséquente par le ligand homologue. Il joue donc un rôle dans la désensibilisation immédiate. La régulation peut être hétérologue, ainsi la cholécystokinine diminue les récepteurs de surface à l'EGF et à l'IGF 2, et le carbachol diminue la densité des récepteurs à la CCK dans les acini [33]. Au niveau des îlots de Langerhans, ce sont les substrats, et en particulier le glucose, qui modifient l'expression des récepteurs de surface ; ainsi les récepteurs à la somatostatine de la cellule $\beta$ augmentent sous fortes concentrations de glucose, ces récepteurs pouvant être en partie localisés à la surface externe de l'enveloppe des granules d'insuline. Le devenir du ligand et du récepteur internalisé est variable. Le ligand peut être dissocié dans les vésicules d'internalisation, puis dégradé comme dans le cas du VIP, de la CCK, de la bombésine, alors que leur récepteur est recyclé ou internalisé dans un pool intracellulaire. Dans le cas de l'EGF, une libération progressive du ligand intact permettant un nouveau recyclage par la cellule a été observée. Le récepteur peut être dégradé, phénomène associé à la désensibilisation prolongée, parfois qualifiée de down regulation ou recyclé et être à nouveau activé. Dans le cas de l'insuline et de l'EGF, une translocation du ligand vers le noyau a été observée, suggérant la possibilité d'une interaction de l'hormone ou de son récepteur avec la chromatine, fait actuellement non prouvé.

Les mécanismes qui règlent la vitesse de synthèse et le cheminement des récepteurs dans la cellule restent encore inconnus. Les stéroïdes peuvent être impliqués : par exemple, les glucocorticoïdes augmentent les récepteurs à la CCK alors qu'ils sont sans effet sur les récepteurs à la bombésine dans les cellules pancréatiques acinaires AR42J. Dans les cultures primaires d'hépatocytes de rat, 


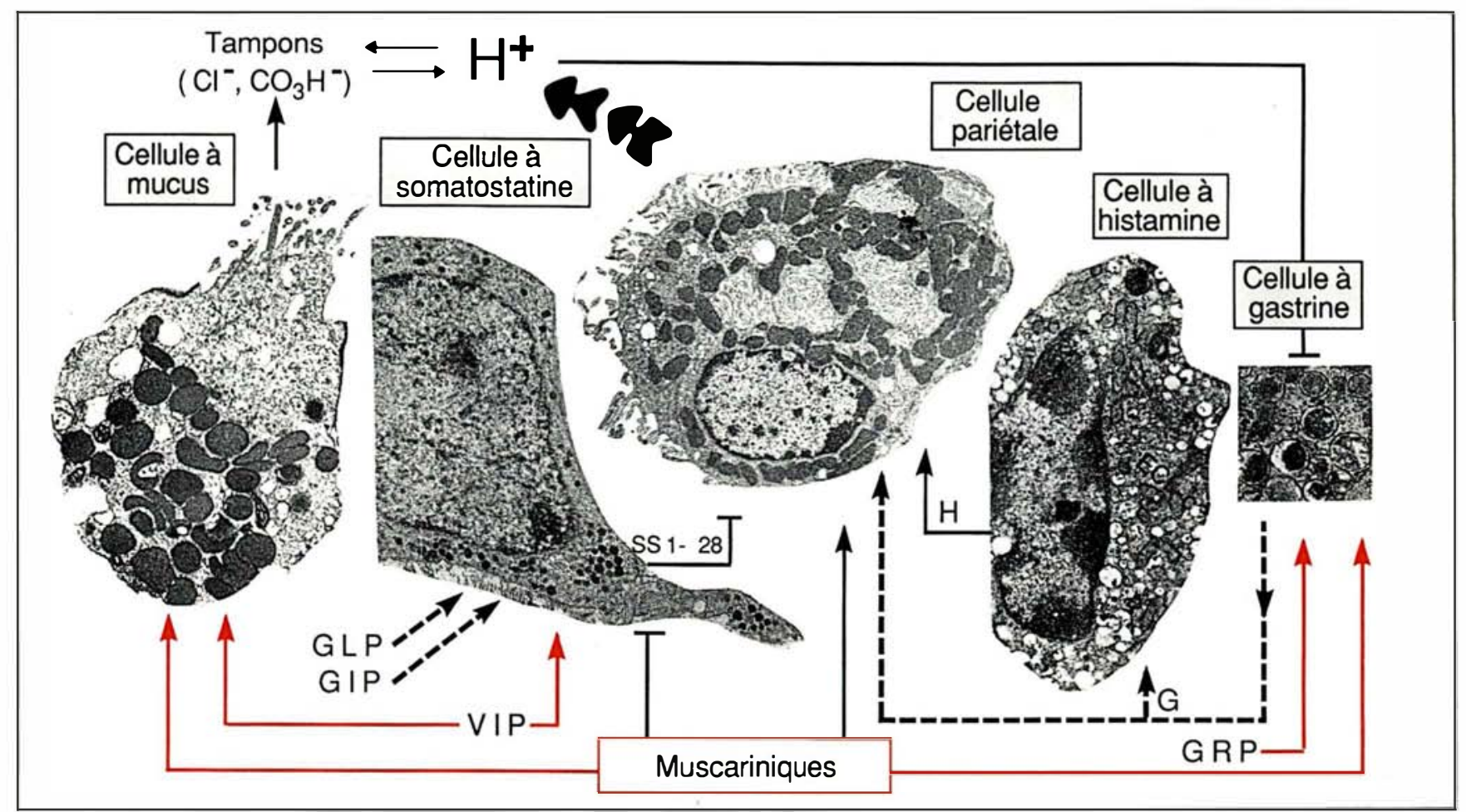

Figure 2. Organisation structurale de la régulation de la fonction acide de l'estomac. Les effets des différents types de cellules de l'épithelium gastrique sont schématisés. Les effets des neuromédiateurs sont indiqués par un trait rouge continu, ceux des hormones par un trait noir discontinu et les effets paracrines par un trait noir continu. Les effets stimulants sont symbolisés par $\rightarrow$ et les effets inhibiteurs par $\rightarrow$. La sécrétion acide $\left(\mathrm{H}^{+}\right)$est tamponnée à des degrés variables par les sécrétions de $\mathrm{CO}_{3} \mathrm{H}^{-}$(venant de cellules duodénales et aussi gastriques) et de mucus comme indiqué par $\underset{2}{2}$. On distingue ainsi les régulations portant sur la sécrétion acide «offensive» et sur les sécrétions «protectrices» pour la muqueuse. La fonction acide est initiée lors de la phase dite “céphalique » de la digestion par l'intermédiaire des neuromédiateurs muscariniques sécrétés par le nerf vague. Outre leur action propre sur la cellule pariétale, ils stimulent la sécrétion de gastrine (G) et diminuent celle de la somatostatine (SS 1-28). Dans la phase «digestive» la sécrétion acide est maintenue du fait de l'effet direct des aliments sur les cellules à sécrétion endocrine et paracrine. A la phase tardive de la digestion, la sécrétion acide est inhibée sous l'influence de divers facteurs neuroendocriniens dénommés entérogastrone quand il s'agit d'hormones provenant de l'intestin. Des deux GLP seul l'effet du GLPI a été testé dans le laboratoire (Hansen, Gespach, Holst, Rosselin à paraître). L'effet des autres peptides inhibiteurs de la fonction acide(EGF), peptide en rapport avec le gène de la calcitonine (CGRP) ainsi que l'effet inhibiteur des prostaglandines ne sont pas représentés sur ce schéma. $H=$ histamine; ECL-cell = cellule à histamine.

les glucocorticoïdes augmentent la liaison de l'EGF tandis que l'insuline s'oppose à cet effet.

\section{Organisation structurale des cellules et réception des signaux}

Les cellules de l'épithélium digestif sont polarisées entre l'axe neurovasculaire et le lumen : les acini pancréatiques autour des canaux ductaux et les cellules hépatiques entre l'endothélium sinusoïdal et les canaux biliaires. Les récepteurs de régulation se trouvent au niveau des membranes basales (ou baso-latérales) qui ont un rôle-clé dans les échanges rapides et massifs entre milieu extra-cellulaire et cellule, ainsi que dans le contrôle de l'adhésion cellulaire. De plus l'or$\mathrm{m} / \mathrm{s} n^{\circ} 5$ vol. 4, mai 88 ganisation cellulaire permet la transmission des signaux neuroendocrines de cellule à cellule : (1) au travers des canaux de communication jonctionnelle (gap junctions) (voir $\mathrm{m} / \mathrm{s} n^{\circ} 9$, vol. 3, p. 550) perméables aux messagers des peptides ayant un faible poids moléculaire, qu'ils soient stimulateurs ou inhibiteurs [34] ; (2) au travers des récepteurs de surface spécifiques intégrant les signaux en provenance des sécrétions autocrines ou paracrines des cellules. Certaines configurations tirent leur intérêt de la juxtaposition de cellules fonctionnellement différentes : ainsi le système réglant l'acidité gastrique comprend à côté de la cellule pariétale acido-sécrétante des cellules à histamine et à somatostatine dont les sécrétions respectivement stimulent ou inhibent la sortie d'ions $\mathrm{H}^{+}$(figure 2). L'organisation cellulaire des îlots de Langerhans est un autre exemple de régulation paracrine comportant autour des cellules $\beta$, des cellules $A$ ou $\mathrm{D}$ dont les sécrétions stimulent (glucagon) ou inhibent (somatostatine) la sécrétion d'insuline sous l'influence du glucose, l'insuline sécrétée diminuant les sécrétions de somatostatine ou de glucagon. La présence de ces communication intercellulaires expliquent les différences notables rencontrées dans l'analyse fonctionnelle des récepteurs sur cellules isolées (perdant leur polarité), sur des cultures organotypiques ou sur organes in situ.

- La disposition des organes par rapport à la circulation (figure $3 a$ ) modifie également la nature ou la 
Figure 3. Organisation structurale de la régulation des fonctions pancréatiques endocrine et exocrine.

\begin{abstract}
A. Connexion vasculaire du bloc duodéno-pancréatique / $d$ 'après Rouvière). L'ensemble du sang veineux gastro-intestinal avec les hormones digestives qu'il contient est drainé par la veine porte qui va au foie. Les hormones restant dans la circulation générale après le passage hépatique atteignent le pancréas par voie artérielle. Les ilots pancréatiques sécrétent leurs hormones dans un système capillaire qui irrigue ensuite le pancréas exocrine, puis le foie par la veine porte, enfin les autres organes et en particulier le tube digestif par la circulation générale.
\end{abstract}

B. Régulation cellulaire. Les cellules cibles indiquées sont celles à bordure en brosse (entérocyte) du duodénum, les cellules acinaires et ductales du pancréas exocrine dont une en mitose (à droite), et les cellules hépatiques (en haut) avec leur espace porte. Les effets des neuromédiateurs sont indiqués en rouge, ceux des hormones en noir discontinu, soit - - - (hormones étant passées par le foie) ou ... (effet direct) et les effets paracrines en noir continu. Les effets stimulants sont indiqués par $\rightarrow$ et les effets inhibiteurs par -1 . Les fonctions du pancréas endocrine et exocrine sont déclenchées dans la phase céphalique de la digestion par l'intermédiaire des récepteurs muscariniques et sans doute du VIP. Elles sont maintenues et ajustées par l'effet direct et sélectif des aliments sur les cellules endocrines disséminées dans le duodénum. Les sécrétions hormonales des cellules $K, I, S$ sont indiquées dans le tableau I. Les cellules $A, B$ et $D$ des îlots du pancréas endocrine présentent une structure facilitant les interactions de type paracrine. Noter, la duplication de la commande nerveuse par la commande hormonale: la stimulation vagale et la CCK activent la sécrétion d'enzyme, la sécrétine et le VIP stimulent la sécrétion de bicarbonates. La stimulation vagale, le VIP et le GIP sti-

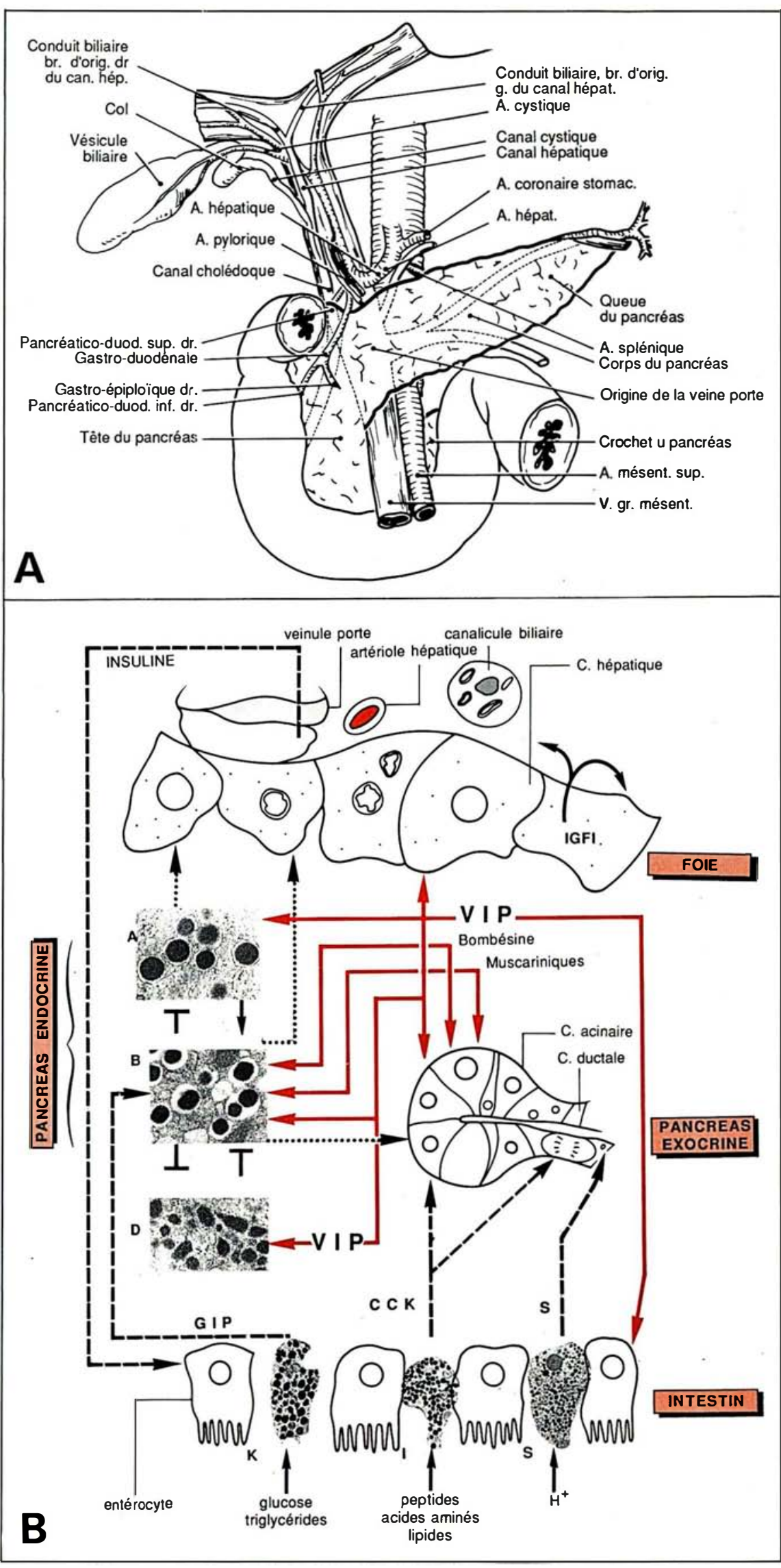

$\mathrm{m} / \mathrm{s} n^{\circ} 5$ vol. 4, mai 8 


\begin{tabular}{|l|}
\hline \multicolumn{1}{|c|}{ Tableau III } \\
EFFET DES PND PRÉSENTS DANS LE \\
CERVEAU SUR LA PRISE ALIMENTAIRE \\
\hline Diminution de la prise alimentaire \\
\hline $\begin{array}{l}\text { Bombésine, GRP } \\
\text { CCK-PZ } \\
\text { Glucagon } \\
\text { Insuline } \\
\text { Somatostatine }\end{array}$ \\
\hline $\begin{array}{l}\text { Augmentation de la prise } \\
\text { alimentaire }\end{array}$ \\
\hline $\begin{array}{l}\text { Polypeptide pancréatique } \\
\text { Galanine } \\
\text { NPY } \\
\text { PYY }\end{array}$ \\
\hline
\end{tabular}

Le neuropeptide $Y$ (NPY) d'origine neuronale et le peptide $Y Y$ d'origine surtout endocrine sont des analogues naturels qui présentent des réactions croisées au niveau de leurs récepteurs spécifiques.

concentration des ligands susceptibles d'atteindre les récepteurs : ainsi les récepteurs de l'insuline au niveau du foie et des acini pancréatiques (figure $3 b$ ) reçoivent des îlots de Langerhans respectivement par un système portal et capillaire, des concentrations d'insuline dix fois plus importantes que celles rencontrées par les cellules en contact avec la circulation périphérique. De telles concentrations sont en particulier susceptibles d'activer des récepteurs type IGFI et ainsi de privilégier l'effet de croissance par rapport à l'effet métabolique de l'hormone [35]. Inversement, le foie en dégradant toute une série de neuropeptides digestifs ne laissera accéder aux tissus périphériques que certains d'entre eux : l'extraction de glucagon, d'insuline et de VIP par le foie est respectivement de 30,50 et $100 \%$. La somatostatine-14 est beaucoup plus rapidement métabolisée que la somatostaine-28 et celle-ci est en partie convertie en somatostatine-14 par une endopeptidase hépatique, fait contribuant à privilégier le rôle hormonal de la somatostatine d'origine digestive par rapport à celle provenant de la cellule $D$ des îlots. Les gastrines, type G-34, G-17, G-14 et la CCK-33 traversent le foie sans dégradation significative alors que l'hexapeptide carboxy-terminal de la gastrine et la CCK-8 sont inactivés (cité dans Rosselin [9]).

$\mathrm{m} / \mathrm{s} n^{\circ} 5$ vol. 4, mai 88
- Enfin, faut-il rappeler que les peptides neurodigestifs ne traversent pas la barrière cérébro-méningée et qu'ainsi ils ne peuvent atteindre leur récepteur spécifique cérébral si ce n'est en dehors de zones très limitées, en particulier hypothalamique. Ainsi les récepteurs des peptides neurodigestifs au niveau du système nerveux central sont activés par ceux de ces peptides qui sont également produits par les cellules nerveuses, tels les peptides de la famille de la CCK-PZ, de la bombésine, du glucagon, du NPY (neuropeptide Y analogue du PYY), des encéphalines [1]. Leurs effets sur l'appétit sont indiqués sur le Tableau III.

\section{Conclusion}

Les cellules périphériques du système digestif et entéro-pancréatique possèdent un remarquable ensemble de récepteurs spécifiques des PND indiquant que leur régulation s'opère aussi bien par voie autocrine et paracrine qu'endocrine et neurocrine. Grâce à ces contrôles la fonction de ces cellules est modifiée en accord avec le rythme, la quantité et la nature de l'apport alimentaire ainsi qu'en fonction des dépenses énergétiques. La fonction de ces cellules est adaptée à la variation des besoins en nutriments induite par la croissance, la grossesse et la lactation. Le développement considérable des recherches sur les récepteurs des peptides neurodigestifs permet maintenant, du moins en partie, l'analyse de la régulation multihormonale des cellules en terme d'interaction spécifique et fournit une base solide pour la compréhension des processus directs d'activation et d'inactivation rendus nécessaires par la régulation de la fonction nutritionnelle. Les connaissances concernant les rapports entre l'activation des récepteurs et la régulation des proto-oncogènes sont beaucoup plus restreintes et le mécanisme d'action des facteurs de croissance sur les cellules digestives reste encore inconnu. Les nouvelles techniques de recherche biologique permettront l'organisation de ces problèmes dans des systèmes expérimentaux appropriés et conduiront à mieux comprendre les mécanismes de régénération des cellules digestives et leur dérèglement

\section{Summary}

The cells of the digestive tract possess a remarkable array of specific receptors for neurodigestive peptides indicating that the regulation of nutritional functions occurs at autocrine, endocrine and neuroendocrine steps. The multipeptidergic regulation of the cells is analyzed in terms of site-site interactions to provide a solid basis for understanding the direct activation-inactivation processes involved in cellular functions and in the cell growth. The physiological relevance of the receptor mapping is shown in relation to the specific pattern of receptor distribution in differentiated digestive cells and to the structural organization of (l) the gastric mucosa, (2) the hepatopancreas and the enteroinsular axis. From those studies it appears that the repartition of the regulatory receptors in digestive tract is teleologically interesting; for example the stimulation of cholinergic and VIPergic receptors and the vagally induced inhibition of somatostatine release act on receptors present in both parietal and beta cells and are involved in the cephalic phase of acid secretion by stomach and insulin secretion by endocrine pancreas. On the other hand, the activity of intestinal epithelium for which no cephalic phase is described appears essentially coordinated by receptors of neuromediators released by the intrinsic neurones. The condition of the neural release is well appropriated to the involvement of different parts of the gut by successive stimulations. Furthermore the step to step involvement of the gut wall in the progression of alimentary bols is associated to a progressive secretion of hormone by the endocrine cells scattered throughout the gut epithelium. Thus the receptors of the insulin-secretory cells are successively triggered by GIP and perhaps CCK from the duodenum, thereafter by oxyntomodulin and GLP I secreted by the terminal part of gut. Regulatory peptides from gut do not cross the blood-brain barrier. Receptors of neurodigestive peptide in brain are triggered by brain hormone regulatory peptides similar to those present in gut and were demonstrated to be involved in the regulation of the food intake. 